ARTICLE

\title{
New Features of the Particle and Heavy Ion Transport Code System; PHITS
}

\author{
Koji NIITA $^{1, *}$, Tatsuhiko SATO ${ }^{2}$,Yosuke IWAMOTO ${ }^{2}$, Norihiro MATSUDA ${ }^{2}$, \\ Hiroshi IWASE $^{3}$, Lembit SIHVER ${ }^{4,5}$, Yukio SAKAMOTO ${ }^{2}$ and Hiroshi NAKASHIMA ${ }^{2}$ \\ ${ }^{1}$ Research Organization for Information Science and Technology, 2-4 Shirakata, Tokai-mura, Naka-gun, Ibaraki-ken, 319-1195, Japan \\ ${ }^{2}$ Japan Atomic Energy Agency, 2-4 Shirakata, Tokai-mura, Naka-gun, Ibaraki-ken, 319-1195, Japan \\ ${ }^{3}$ KEK, 1-1 Oho, Tsukuba, Ibaraki-ken, 305-0801, Japan \\ ${ }^{4}$ Chalmaers University of Technology, Goetheborg, Sweden \\ ${ }^{5}$ Roanoke College, VA, USA
}

\begin{abstract}
We present recent developments of the PHITS code concerned with the event generator mode for low energy neutron transport, the function for correlated sources and micro-dosimetric treatment of the deposit energy. One of the recent important issues which general-purpose transport codes such as PHITS should treat is "correlations". We have therefore developed a new treatment of radiation behaviour for the low energy neutron transport by introducing an event generator model, in which we have combined the nuclear data and a theoretical model so as to trace all correlations among ejectiles keeping the energy and momentum conservation in a collision. In addition to the event generator mode, we discuss a role of the correlated source, which is newly introduced in PHITS, for the correlated observables in event-by-event. We also present a new tally function SED (Specific Energy Distribution), which enables us to estimate the deposit energy distribution in a micro-dosimetric region such as a cell or DNA..
\end{abstract}

KEYWORDS: PHITS, event generator mode, correlated source, specific energy distribution

\section{Introduction}

The PHITS code, ${ }^{1)}$ has been used for various research fields such as radiation science, accelerator and its shielding design, space research, medical application, and material research. Further developments of PHITS are actively performed by the collaboration among RIST, JAEA, KEK, and Chalmers University. In this paper, we present recent developments of PHITS concerned with the event generator mode for low energy neutron transport (which indicates the energy range below $20 \mathrm{MeV}$ down to thermal energy in this paper), the function for correlated sources, and micro-dosimeric treatment of the deposit energy. One of the recent important issues which general-purpose transport codes such as PHITS should treat is "correlations". A typical example for such a correlated quantity is the deposit energy distribution in a cell, which is necessary to estimate the response function of detectors, the single event upset probability of semiconductor memory cells and the biological dose based on microdosimetry. We have therefore developed a new treatment of radiation behaviour for the low energy neutron transport by introducing an event generator model, in which we have combined the nuclear data and a theoretical model so as to trace all correlations among ejectiles keeping the energy and momentum conservation in a collision. In addition to the event generator mode, we discuss a role of the correlated source, which is newly introduced in PHITS, for the correlated observables in event-by-event. We also present a new tally function SED (Specific Energy Distribution), which

*Corresponding author, E-mail:niita@tokai.rist.or.jp

(C) 2011 Atomic Energy Society of Japan, All Rights Reserved. enables us to estimate the deposit energy distribution in a micro-dosimetric region such as a cell or DNA.

\section{Event Generator Mode}

For high energy transport calculations, the nuclear reaction models are commonly used instead of the evaluated nuclear data to describe the ejectiles from each nuclear reaction, since there is no enough evaluated nuclear data for the high energy reactions. The reaction models usually generate each event with keeping the energy and momentum by the Monte Carlo method. In this sense, the reaction model is called as "Event Generator". We can then extract any information from the transport calculations with such reaction models. On the other hand, the transport calculations based on the Boltzmann equation with the nuclear data, e.g. low energy neutron transport calculations, have no such concept of "Event". For the actual numerical solution of the Boltzmann equation, one usually employs the test particle method in which the one-body phase-space distribution function is evaluated by integrating the test particle tracks in the phase-space. In this numerical procedure, things are going on in a very similar manner as in the high energy transport calculation with the event generator. However, there is no "Event" in this type of calculations. One history in this type of calculation has no physical meaning but gives us only a statistical weight. The observables are obtained after averaged out the statistical weight. There is no physical meaning in the distribution around the mean value but only the statistical variance. However, the distribution around the mean value calculated in the high energy transport code with the 


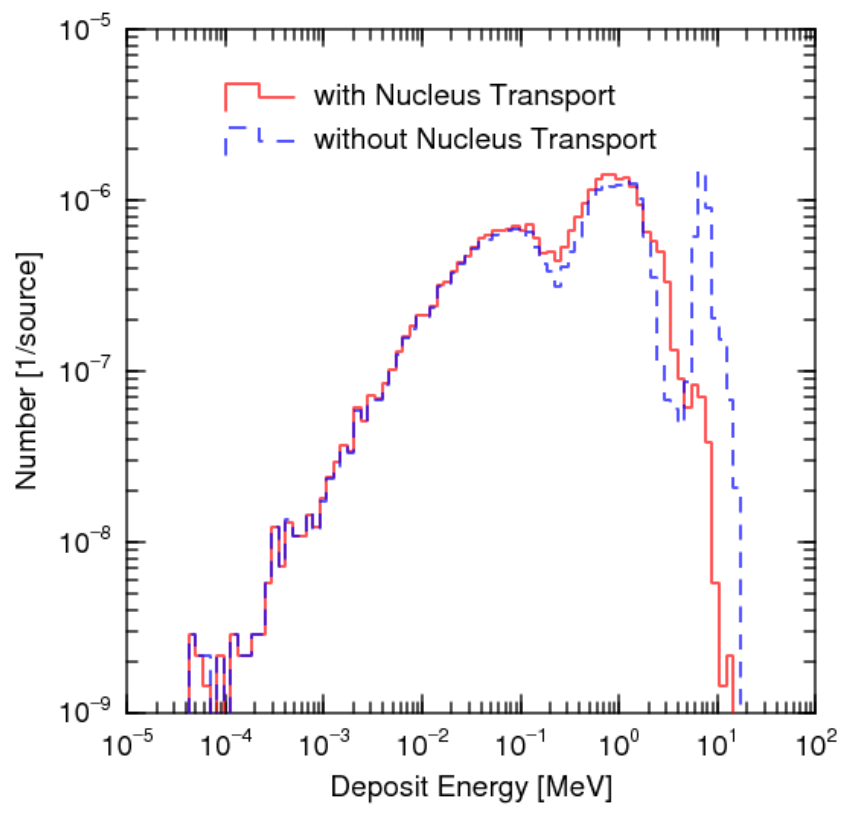

Fig. 1 Deposit energy distribution in a thin $(3 \mu \mathrm{m})$ Si tip irradiated by mono energetic neutron at $19 \mathrm{MeV}$. The solid (red) line is the result of the event generator mode of PHITS with nucleus transport, and the dashed (blue) line is the result by a local approximation, i.e. without nucleus transport.

event generator mode is a real physical quantity, which is often required as mentioned above.

One possibility to construct an event for low energy neutron collision might be to make a pure theoretical model to describe the collision. However, it is almost hopeless since the cross section of neutron in the low energy below $20 \mathrm{MeV}$ has a lot of resonance structure and all of the resonances cannot be described purely by a theoretical model. Therefore, we have developed a new treatment of collisional processes in PHITS for low energy neutrons by combining the evaluated nuclear data and a theoretical model. The detail of the event generator model in PHITS and its validation are presented in another paper of this issue. ${ }^{2)}$

By the event generator mode in PHITS for low energy neutron transport phenomena, we can calculate many new quantities which cannot be obtained directly by the nuclear data calculation, e.g. kinetic energy distribution of residual nuclei, charged particles, and two-particle correlation. To get these quantities by the nuclear data calculation, one needs additionally evaluated data, for example, the KERMA factor, DPA values, PKA spectra and so on. Here we show an example calculated by the event generator model by using only the neutron cross sections of the nuclear data.

The deposit energy distribution in a cell is a typical quantity beyond one-body observable. This quantity is well known as a pulse height tally in the MCNP code. ${ }^{3)}$ However, this is a conceptually wrong quantity to be treated in the MCNP-type calculation based on the one-body Boltzmann equation for the one-body phase-space distribution function. As mentioned in the MCNP manual, this tally is very restricted to use for neutron transport in MCNP. Namely, this tally does not work if a collision produces multiple neutrons.

Figure 1 shows the deposit energy distribution in a thin Si tip irradiated by neutron with mono energetic neutron at $19 \mathrm{MeV}$. The thickness of the Si tip is $3 \mu \mathrm{m}$. If we calculate the deposit energy by using the KERMA factor in MCNP-type calculation, we get a delta-function-like single peak at $100 \mathrm{eV}$ with almost no distribution, since the KERMA factor is the average kinetic energy of the charged particles and recoil nuclei. However, Fig. 1 shows broad distribution obtained by the event generator model in PHITS, where the deposit energy is estimated from the ionization losses of the charged particles and the recoil nuclei. This distribution is not due to a statistical fluctuation in the Monte Carlo numerical calculation but a physical quantity to be considered. This distribution is also very important to estimate the single event upset probability of semiconductor memory cells, since only the events which induce the deposit energy greater than a certain threshold energy contribute to the error.

In Fig. 1, we have plotted two distributions, solid line and dashed line. The dashed line was obtained by the local approximation (KERMA approximation), in which all charged particles and recoil nuclei produced in neutron induced reactions are assumed to be stopped and deposit their kinetic energy at the collision point. This approximation is valid under the condition where the range of charged particles is much smaller than the size of the system. For this example, the both are comparable, since the range of charged particles is of the order of $\mu \mathrm{m}$, while the thickness of $\mathrm{Si}$ is $3 \mu \mathrm{m}$. Therefore we transported the charged particles and the recoil nuclei in the PHITS calculation without the local approximation. The result is shown by the solid line in Fig. 1 . This shows that the highest energy peak of the dashed line is disappeared. This suggests that some part of the charged particles or the recoil nuclei can escape from Si tip to the outside. For this case, we found that alpha particles mainly contribute to the highest energy peak, while the second peak around $1 \mathrm{MeV}$ mainly comes from the recoil of $\mathrm{Al}$, the third peak around $0.1 \mathrm{MeV}$ from the recoil of the Si nuclei.

This example indicates two important points. The first is that the deposit energy distribution in a cell is described in a more realistic way by the event generator model in PHITS even for low energy neutron transport below $20 \mathrm{MeV}$. The second is that the local approximation is not valid for the system which size is comparable to the range of the transport charged particles and recoil nuclei.

\section{Correlated Source}

In the PHITS code, many source functions have been already installed. In these, one of the nice functions is the "multi-source", by which user can define complicated sources consisted of different kind of source particles at different source positions with different energy spectra in an easy way. The PHITS code generates the source particles according to these distributions by the Monte Carlo method. In this "multi-source", therefore, each source particle has no correlation with the other source particles. However, the 
correlations between the source particles are sometimes required. A typical example of the correlated source is the neutrons emitted from a fission event. Some neutrons are emitted from one fission event of fissile nuclei. Usually one uses the information of the energy spectrum and multiplicity of emitted neutrons from fission events as a source of the calculation. If one wants to see the one-body observables, e.g. the average flux, heat, and so on, from the calculations, there is no problem. But if one wants to see higher order observables; deposit energy distribution, two particles correlated flux, coincident events, it is not enough. One needs correlated event-by-event information which cannot be described by using uncorrelated sources. Therefore we have introduced the function in which we generate the source particles according to the correlation function defined by user and treat them in the same event. By this new function, one can investigate the differences of the flux and deposit energy around the fissile nuclei caused by the uncorrelated neutron source or by the correlated neutron from fission events. Another example of the correlated source is the charged particles and ions produced by laser-driven ion accelerator. When we describe the transport phenomena starting from the charged particle and ions produced by laser-driven ion accelerator, we use the information of the experimental data of produced charged particles as a source. These charged particles are correlated within an event caused by one laser shot. If one wants to estimate the correlated observables in event-by-event, one should also treat the correlated source.

\section{Specific Energy Distribution}

For the ionization process of the charged particles and nuclei, we have used in the PHITS code the macroscopic model, continuous-slowing-down approximation, to estimate the stopping power $d E / d x$, the angle straggling and energy straggling around the average energy loss according to the charge density and velocity. In order to investigate the radiation effects on biological cells in a microscopic way, it is important to estimate the probability density of deposit energies in microscopic sites, which are called lineal energy $y$ or special energy $z$. This is also an important quantity for the estimation of the relative biological effectiveness (RBE) of charged particles. For this, we need a microscopic model of the ionization processes in which each track structure of the charged particles is treated explicitly. However, it is not a clever way to connect such microscopic track simulation code to the transport code for macroscopic scale such as PHITS. We have, therefore, introduced a special tally named [T-SED] (Tally for Specific Energy Distribution) for calculating the microscopic probability densities using the mathematical functions that can instantaneously give the probability density of deposit energies around the trajectory of charged particles. The mathematical functions have been developed on the basis of the track structure simulation, considering the productions of $\delta$-rays and Auger electrons. The details of the derivation are given in the references. ${ }^{4,5)}$

Using this tally, we can get the information of the probability densities of $y$ and $z$ basically in water. Though one can also calculate the probability densities in different materials, the accuracy has not been checked yet in other materials. If you use this tally with the Event Generator mode of PHITS mentioned above, you can obtain the probability densities of $y$ and $z$ caused by low energy neutron transport in the same manner as in the charged particles. As an example of the application of this tally, we have calculated the biological dose for charged particle therapy using this tally coupled to the microdosimetric kinetic (MK) model. ${ }^{6)}$ In the MK model, the RBE of charged particles can be determined from the probability densities of $y$ in tumor, which can be calculated by the SED tally of PHITS within reasonable computational time. The results of the biological dose estimated by this method agree in general within $20 \%$ error with the several experimental data obtained by irradiating a slab phantom by the spread-out Bragg peak beams as well as several mono energetic heavy ions up to $400 \mathrm{MeV} / \mathrm{n}^{5}$ )

\section{Summary}

We have presented new features of the PHITS code, (1) Event Generator mode, (2) Correlated source, (3) Specific Energy Distribution tally. The first two things are related to "correlation", which is one of the recent important issues of general purpose transport codes. By these features, we can estimate new quantities beyond one-body observables, the deposit energy distribution, coincident quantities, and event-by-event observables, which cannot be obtained by the transport calculations based on the Boltzmann equation with the evaluated nuclear data. The last one is related to microscopic treatment of charged particles in materials. We have developed a mathematical model for calculating the microdosimetric quantity $y$ around the trajectory of charged particles on the basis of track-structure simulation, and implement it as a new tally function into the PHITS code in order to estimate the probability density of $y$ in macroscopic matters. By these new features of the PHITS code, we expect that the PHITS code will be applied to the analysis of many problems in broad and new application fields in future.

\section{References}

1) H. Iwase, K. Niita and T. Nakamura, J. Nucl. Sci. Technol., 39, 1142-1151 (2002); K. Niita, T. Sato, H. Iwase, H. Nose, H. Nakashima, L. Sihver, "PHITS - a particle and heavy ion transport code system,” Radiat. Meas., 41, 1080-1090 (2006).

2) Y. Iwamoto, K. Niita, T. Sato, N. Matsuda, H. Iwase, H. Nakashima, Y. Sakamoto, "Validation of Event Generator Model in the PHITS Code for the Low Energy Neutron-Induced Reaction," in this issue.

3) J. F. Briesmeister, (Ed.), MCNP - A General Monte Carlo $N$-Particle Transport Code - Version 4C, LA13709-M, Los Alamos National Laboratory (LANL) (2000).

4) T. Sato, R. Watanabe, K. Niita, "Development of a calculation method for estimating the specific energy distribution in complex radiation fields,” Radiat. Prot. Dosim., 122, 41-45 (2006).

5) T. Sato, Y. Kase, R. Watanabe, K. Niita, L. Sihver, "Biological dose estimation for charged-particle therapy using an improved PHITS code coupled with a microdosimetric kinetic model," Radiat. Res., 171, 107-117 (2009). 
6) R. B. Hawkins, “A microdosimetric-kinetic model of cell death from exposure to ionizing radiation of any LET, with experi- mental and clinical applications,” Int. J. Radiat. Biol., 69, 739-755 (1996). 